\title{
Multiple solutions for biharmonic equations with improved subcritical polynomial growth and subcritical exponential growth
}

Ruichang Pei ${ }^{1,2^{*}}$ and Jihui Zhang ${ }^{2}$

"Correspondence: prc211@163.com
${ }^{1}$ School of Mathematics and
Statistics, Tianshui Normal
University, Tianshui, 741001,
P.R. China
${ }^{2}$ School of Mathematical Sciences,
Nanjing Normal University, Nanjing,
210097, P.R. China

"Correspondence: prc211@163.com University, Tianshui, 741001

\begin{abstract}
The main purpose of this paper is to establish the existence of three nontrivial solutions for a class of fourth-order elliptic equations with subcritical polynomial growth and subcritical exponential growth by using the minimax method and Morse theory.
\end{abstract}

Keywords: Morse theory; Adams-type inequality; subcritical polynomial growth; subcritical exponential growth

\section{Introduction}

Consider the following Navier boundary value problem:

$$
\left\{\begin{array}{l}
\Delta^{2} u+c \Delta u=f(x, u), \quad \text { in } \Omega \\
u=\Delta u=0, \quad \text { in } \partial \Omega
\end{array}\right.
$$

where $\Delta^{2}$ is the biharmonic operator and $\Omega$ is a bounded smooth domain in $\mathbb{R}^{N}(N \geq 4)$. In problem (1), let $f(x, u)=b\left[(u+1)^{+}-1\right]$, then we get the following Dirichlet problem:

$$
\left\{\begin{array}{l}
\Delta^{2} u+c \Delta u=b\left[(u+1)^{+}-1\right], \quad \text { in } \Omega \\
u=\Delta u=0, \quad \text { in } \partial \Omega
\end{array}\right.
$$

where $u^{+}=\max \{u, 0\}$ and $b \in \mathbb{R}$. We let $\lambda_{k}(k=1,2, \ldots)$ denote the eigenvalues of $-\Delta$ in $H_{0}^{1}(\Omega)$.

Fourth-order problems of this class with $N>4$ have been studied by many authors. In [1], Lazer and Mckenna pointed out that this type of nonlinearity provides a model to study traveling waves in suspension bridges. Since then, more general nonlinear fourth-order elliptic boundary value problems have been studied. For problem (2), Lazer and Mckenna [2] proved the existence of $2 k-1$ solutions when $N=1$, and $b>\lambda_{k}\left(\lambda_{k}-c\right)$ by the global bifurcation method. In [3], Tarantello found a negative solution when $b \geq \lambda_{1}\left(\lambda_{1}-c\right)$ by a degree argument. For problem (1) when $f(x, u)=b g(x, u)$, Micheletti and Pistoia [4] proved

(c) 2015 Pei and Zhang. This article is distributed under the terms of the Creative Commons Attribution 4.0 International License (http://creativecommons.org/licenses/by/4.0/), which permits unrestricted use, distribution, and reproduction in any medium, provided you give appropriate credit to the original author(s) and the source, provide a link to the Creative Commons license, and indicate if changes were made. 
that there exist two or three solutions for a more general nonlinearity $g$ by the variational method. Xu and Zhang [5] discussed the problem when $f$ satisfies the local superlinearity and sublinearity. Zhang [6] proved the existence of solutions for a more general nonlinearity $f(x, u)$ under some weaker assumptions. Zhang and Li [7] proved the existence of multiple nontrivial solutions by means of Morse theory and local linking. An and Liu [8] and Liu and Wang [9] also obtained the existence result for nontrivial solutions when $f$ is asymptotically linear at positive infinity. In [10], Zhang and Wei obtained the existence of infinitely many solutions when the nonlinearity involves a combination of superlinear and asymptotically linear terms. As far as the problem (1) is concerned, existence results of sign-changing solutions were also obtained (see, e.g., [11, 12]).

We notice that almost all the works (see [4-12]) mentioned above involve the nonlinear term $f(x, u)$ of a subcritical (polynomial) growth, say,

(SCP): there exist positive constants $c_{1}$ and $c_{2}$ and $q_{0} \in\left(1, p^{*}-1\right)$ such that

$$
|f(x, t)| \leq c_{1}+c_{2}|t|^{q_{0}} \quad \text { for all } t \in \mathbb{R} \text { and } x \in \Omega,
$$

where $p^{*}=2 N /(N-4)$ denotes the critical Sobolev exponent. One of the main reasons to assume this condition (SCP) is to use the Sobolev compact embedding $H^{2}(\Omega) \cap H_{0}^{1}(\Omega) \hookrightarrow$ $L^{q}(\Omega)\left(1 \leq q<p^{*}\right)$. In that case, it is easy to see that seeking weak solutions of problem (1) is equivalent to finding nonzero critical points of the following functional on $H^{2}(\Omega) \cap H_{0}^{1}(\Omega)$ :

$$
I(u)=\frac{1}{2} \int_{\Omega}\left(|\Delta u|^{2}-c|\nabla u|^{2}\right) d x-\int_{\Omega} F(x, u) d x,
$$

where $F(x, u)=\int_{0}^{u} f(x, t) d t$. In this paper, inspired by Lam and Lu [13], our first main result will be to study problem (1) in the improved subcritical polynomial growth

(SCPI): $\lim _{t \rightarrow \infty} \frac{f(x, t)}{|t|^{p^{*}-1}}=0 \quad$ uniformly on $x \in \Omega$,

which is weaker than (SCP). Note that, in this case, we do not have the Sobolev compact embedding anymore. Our work is to study problem (1) when nonlinearity $f$ does not satisfy the Ambrosetti-Rabinowitz condition, i.e., for some $\theta>2$ and $\gamma>0$,

$$
0<\theta F(x, t) \leq f(x, t) t \quad \text { for all }|t| \geq \gamma \text { and } x \in \Omega \text {. }
$$

In fact, this condition was studied by Liu and Wang in [14] in the case of Laplacian by the Nehari manifold approach. However, we will use a suitable version of the mountain pass theorem and Morse theory to get three nontrivial solutions for problem (1) in the general case $N>4$. Indeed, in this case, we have obtained two nontrivial solutions for problem (1) in [15] via the mountain pass theorem and a truncated technique.

Let us now state our results. In this paper, we always assume that $f(x, t) \in C^{1}(\bar{\Omega} \times \mathbb{R})$. The conditions imposed on $f(x, t)$ are as follows:

$\left(\mathrm{H}_{1}\right) f(x, t) t \geq 0$ for all $x \in \Omega, t \in \mathbb{R}$;

$\left(\mathrm{H}_{2}\right) \lim _{|t| \rightarrow 0} \frac{f(x, t)}{t}=f_{0}$ uniformly for $x \in \Omega$, where $f_{0}$ is a constant;

$\left(\mathrm{H}_{3}\right) \lim _{|t| \rightarrow \infty} \frac{f(x, t)}{t}=+\infty$ uniformly for $x \in \Omega$;

$\left(\mathrm{H}_{4}\right) \frac{f(x, t)}{|t|}$ is nondecreasing in $t \in \mathbb{R}$ for any $x \in \Omega$. 
Let $0<\mu_{1}$ be the first eigenvalue of $\left(\Delta^{2}-c \Delta, H^{2}(\Omega) \cap H_{0}^{1}(\Omega)\right)\left(c<\lambda_{1}\right)$ and $\varphi_{1}(x)>0$ be the eigenfunction corresponding to $\mu_{1}$. Throughout this paper, we denote by $|\cdot|_{p}$ the $L^{p}(\Omega)$ norm and the norm of $u$ in $H^{2}(\Omega) \cap H_{0}^{1}(\Omega)$ will be defined by

$$
\|u\|:=\left(\int_{\Omega}\left(|\Delta u|^{2}-c|\nabla u|^{2}\right) d x\right)^{\frac{1}{2}}
$$

We also define $E=H^{2}(\Omega) \cap H_{0}^{1}(\Omega)$. In fact, the norm $\|\cdot\|$ is equivalent to another norm $\|\cdot\|_{E}$ defined by

$$
\|u\|_{E}:=\left(\int_{\Omega}|\Delta u|^{2} d x\right)^{\frac{1}{2}}
$$

on $E$, i.e., there exist two positive constants $C^{*}, C^{* *}$ such that

$$
C^{* *}\|u\| \leq\|u\|_{E} \leq C^{*}\|u\|
$$

Theorem 1.1 Let $N>4$ and assume that $f$ has the improved subcritical polynomial growth on $\Omega$ (condition (SCPI)) and satisfies $\left(\mathrm{H}_{1}\right)-\left(\mathrm{H}_{4}\right)$. If $f_{0}<\mu_{1}$, then problem (1) has at least three nontrivial solutions.

In the case of $N=4$, we have $p^{*}=+\infty$. So it is necessary to introduce the definition of the subcritical (exponential) growth in this case. By the improved Adams inequality (see [16]) for the fourth-order derivative, we have

$$
\sup _{u \in E,\|u\|_{E} \leq 1} \int_{\Omega} e^{32 \pi^{2} u^{2}} d x \leq C|\Omega|
$$

where $C$ is a positive constant (see Lemma 2.2). So, we now define the subcritical (exponential) growth in this case as follows:

(SCE): $f$ has subcritical (exponential) growth on $\Omega$, i.e., $\lim _{t \rightarrow \infty} \frac{|f(x, t)|}{\exp \left(\alpha t^{2}\right)}=0$ uniformly on $x \in \Omega$ for all $\alpha>0$.

When $N=4$ and $f$ has the subcritical (exponential) growth (SCE), our work is still to study problem (1) without the (AR) condition. Our result is as follows.

Theorem 1.2 Let $N=4$ and assume that $f$ has the subcritical exponential growth on $\Omega$ (condition (SCE)) and satisfies $\left(\mathrm{H}_{1}\right)-\left(\mathrm{H}_{4}\right)$. If $f_{0}<\mu_{1}$, then problem (1) has at least three nontrivial solutions.

Remark 1.1 Indeed, in this case we have already obtained two nontrivial solutions for problem (1) in [15] via the mountain pass theorem and a truncated technique. So, this paper is a completion for our previous work (see [15]).

\section{Preliminaries and auxiliary lemmas}

Definition 2.1 Let $\left(E,\|\cdot\|_{E}\right)$ be a real Banach space with its dual space $\left(E^{*},\|\cdot\|_{E^{*}}\right)$ and $I \in C^{1}(E, \mathbb{R})$. For $c \in \mathbb{R}$, we say that $I$ satisfies the $(C)_{c}$ condition (Cerami condition) if for any sequence $\left\{x_{n}\right\} \subset E$ with

$$
I\left(x_{n}\right) \rightarrow c, \quad\left\|D I\left(x_{n}\right)\right\|_{E^{*}}\left(1+\left\|x_{n}\right\|_{E}\right) \rightarrow 0,
$$

there is a subsequence $\left\{x_{n_{k}}\right\}$ such that $\left\{x_{n_{k}}\right\}$ converges strongly in $E$. 
We have the following version of the mountain pass theorem (see [17]).

Proposition 2.1 Let E be a real Banach space and suppose that $I \in C^{1}(E, R)$ satisfies the condition

$$
\max \left\{I(0), I\left(u_{1}\right)\right\} \leq \alpha<\beta \leq \inf _{\|u\|=\rho} I(u)
$$

for some $\alpha<\beta, \rho>0$ and $u_{1} \in E$ with $\left\|u_{1}\right\|>\rho$. Let $c \geq \beta$ be characterized by

$$
c=\inf _{\gamma \in \Gamma} \max _{0 \leq t \leq 1} I(\gamma(t))
$$

where $\Gamma=\left\{\gamma \in C([0,1], E), \gamma(0)=0, \gamma(1)=u_{1}\right\}$ is the set of continuous paths joining 0 and $u_{1}$. Then there exists a sequence $\left\{u_{n}\right\} \subset E$ such that

$$
I\left(u_{n}\right) \rightarrow c \geq \beta \quad \text { and } \quad\left(1+\left\|u_{n}\right\|\right)\left\|I^{\prime}\left(u_{n}\right)\right\|_{E^{*}} \rightarrow 0 \quad \text { as } n \rightarrow \infty
$$

Consider the following problem:

$$
\left\{\begin{array}{l}
\Delta^{2} u+c \Delta u=f_{+}(x, u), \quad x \in \Omega \\
\left.u\right|_{\partial \Omega}=\left.\Delta u\right|_{\partial \Omega}=0
\end{array}\right.
$$

where

$$
f_{+}(x, t)= \begin{cases}f(x, t), & \text { if } t>0 \\ 0, & \text { if } t \leq 0\end{cases}
$$

Define a functional $I_{+}: E \rightarrow \mathbb{R}$ by

$$
I_{+}(u)=\frac{1}{2} \int_{\Omega}\left(|\Delta u|^{2}-c|\nabla u|^{2}\right) d x-\int_{\Omega} F_{+}(x, u) d x
$$

where $F_{+}(x, t)=\int_{0}^{t} f_{+}(x, s) d s$; then $I_{+} \in C^{1}(E, \mathbb{R})$.

Lemma 2.1 Let $N>4$ and $\varphi_{1}>0$ be a $\mu_{1}$-eigenfunction with $\left\|\varphi_{1}\right\|=1$ and assume that $\left(\mathrm{H}_{2}\right),\left(\mathrm{H}_{3}\right)$ and (SCPI) hold. If $f_{0}<\mu_{1}$, then

(i) there exist $\rho, \alpha>0$ such that $I_{+}(u) \geq \alpha$ for all $u \in E$ with $\|u\|=\rho$,

(ii) $I_{+}\left(t \varphi_{1}\right) \rightarrow-\infty$ as $t \rightarrow+\infty$.

Proof By (SCPI), $\left(\mathrm{H}_{2}\right)$ and $\left(\mathrm{H}_{3}\right)$, for any $\varepsilon>0$, there exist $A_{1}=A_{1}(\varepsilon), B_{1}=B_{1}(\varepsilon)$ and $l>2 \mu_{1}$ such that for all $(x, s) \in \Omega \times \mathbb{R}$,

$$
\begin{aligned}
& F_{+}(x, s) \leq \frac{1}{2}\left(f_{0}+\varepsilon\right) s^{2}+A_{1} s^{p^{*}}, \\
& F_{+}(x, s) \geq \frac{1}{2} l s^{2}-B_{1} .
\end{aligned}
$$


Choose $\varepsilon>0$ such that $\left(f_{0}+\varepsilon\right)<\mu_{1}$. By (4), the Poincaré inequality and the Sobolev inequality $|u|_{p^{*}}^{p^{*}} \leq K\|u\|^{p^{*}}$, we get

$$
\begin{aligned}
I_{+}(u) & \geq \frac{1}{2}\|u\|^{2}-\frac{f_{0}+\varepsilon}{2}|u|_{2}^{2}-A_{1}|u|_{p^{*}}^{p^{*}} \\
& \geq \frac{1}{2}\left(1-\frac{f_{0}+\varepsilon}{\mu_{1}}\right)\|u\|^{2}-A_{1} K\|u\|^{p^{*}} .
\end{aligned}
$$

So, part (i) is proved if we choose $\|u\|=\rho>0$ small enough.

On the other hand, from (5) we have

$$
I_{+}\left(t \varphi_{1}\right) \leq \frac{1}{2}\left(1-\frac{l}{\mu_{1}}\right) t^{2}+B_{1}|\Omega| \rightarrow-\infty \quad \text { as } t \rightarrow+\infty
$$

Thus part (ii) is proved.

Lemma 2.2 (see [16]) Let $\Omega \subset \mathbb{R}^{4}$ be a bounded domain. Then there exists a constant $C>0$ such that

$$
\sup _{u \in E,\|u\|_{E} \leq 1} \int_{\Omega} e^{32 \pi^{2} u^{2}} d x \leq C|\Omega|
$$

where $\|u\|_{E}=\left(\int_{\Omega}|\Delta u|^{2} d x\right)^{\frac{1}{2}}$. This inequality is sharp.

Lemma 2.3 Let $N=4$ and $\varphi_{1}>0$ be a $\mu_{1}$-eigenfunction with $\left\|\varphi_{1}\right\|=1$ and assume that $\left(\mathrm{H}_{2}\right),\left(\mathrm{H}_{3}\right)$ and (SCE) hold. If $f_{0}<\mu_{1}$, then

(i) there exist $\rho, \alpha>0$ such that $I_{+}(u) \geq \alpha$ for all $u \in E$ with $\|u\|=\rho$,

(ii) $I_{+}\left(t \varphi_{1}\right) \rightarrow-\infty$ as $t \rightarrow+\infty$.

Proof By (SCE), $\left(\mathrm{H}_{2}\right)$ and $\left(\mathrm{H}_{3}\right)$, for any $\varepsilon>0$, there exist $A_{1}=A_{1}(\varepsilon), B_{1}=B_{1}(\varepsilon), \kappa>0, q>2$ and $l>2 \mu_{1}$ such that for all $(x, s) \in \Omega \times \mathbb{R}$,

$$
\begin{aligned}
& F_{+}(x, s) \leq \frac{1}{2}\left(f_{0}+\varepsilon\right) s^{2}+A_{1} \exp \left(\kappa|s|^{2}\right) s^{q}, \\
& F_{+}(x, s) \geq \frac{1}{2} l s^{2}-B_{1} .
\end{aligned}
$$

Choose $\varepsilon>0$ such that $\left(f_{0}+\varepsilon\right)<\mu_{1}$. By (6), the Hölder inequality and Lemma 2.2, we get

$$
\begin{aligned}
I_{+}(u) & \geq \frac{1}{2}\|u\|^{2}-\frac{f_{0}+\varepsilon}{2}|u|_{2}^{2}-A_{1} \int_{\Omega} \exp \left(\kappa|u|^{2}\right)|u|^{q} d x \\
& \geq \frac{1}{2}\left(1-\frac{f_{0}+\varepsilon}{\mu_{1}}\right)\|u\|^{2}-A_{1}\left(\int_{\Omega} \exp \left(\kappa r\|u\|_{E}^{2}\left(\frac{|u|}{\|u\|_{E}}\right)^{2}\right) d x\right)^{\frac{1}{r}}\left(\int_{\Omega}|u|^{r^{\prime} q} d x\right)^{\frac{1}{r^{\prime}}} \\
& \geq \frac{1}{2}\left(1-\frac{f_{0}+\varepsilon}{\mu_{1}}\right)\|u\|^{2}-C\|u\|^{q},
\end{aligned}
$$

where $r>1$ is sufficiently close to $1,\|u\|_{E} \leq \sigma$ and $\kappa r \sigma^{2}<32 \pi^{2}$. So, part (i) is proved if we choose $\|u\|=\rho>0$ small enough since $\|u\|_{E} \leq C^{*}\|u\|$. 
On the other hand, from (7) we have

$$
I_{+}\left(t \varphi_{1}\right) \leq \frac{1}{2}\left(1-\frac{l}{\mu_{1}}\right)|t|^{2}+B_{1}|\Omega| \rightarrow-\infty \quad \text { as } t \rightarrow+\infty .
$$

Thus part (ii) is proved.

Lemma 2.4 For the functional $I_{+}$, if condition $\left(\mathrm{H}_{4}\right)$ holds, and for any $\left\{u_{n}\right\} \in E$ with

$$
\left\langle I_{+}^{\prime}\left(u_{n}\right), u_{n}\right\rangle \rightarrow 0 \quad \text { as } n \rightarrow \infty
$$

then there is a subsequence, still denoted by $\left\{u_{n}\right\}$, such that

$$
I_{+}\left(t u_{n}\right) \leq \frac{1+t^{2}}{2 n}+I_{+}\left(u_{n}\right) \quad \text { for all } t \in \mathbb{R} \text { and } n \in N .
$$

Proof This lemma is essentially due to [18]. We omit the proof.

Lemma 2.5 Under the assumptions of Theorem 1.1, then $I_{+}$and I satisfy the $(C)_{c}$ condition.

Proof We first do the proof for $I_{+}$. Let $\left\{u_{n}\right\} \subset E$ be a $(C)_{c}(c \in \mathbb{R})$ sequence such that for every $n \in \mathbb{N}$,

$$
\begin{aligned}
& I_{+}\left(u_{n}\right)=\frac{1}{2}\left\|u_{n}\right\|^{2}-\int_{\Omega} F_{+}\left(x, u_{n}\right) d x=c+o(1), \\
& \left(1+\left\|u_{n}\right\|\right)\left\|I_{+}^{\prime}\left(u_{n}\right)\right\|_{E} \rightarrow 0 \quad \text { as } n \rightarrow \infty .
\end{aligned}
$$

Clearly, (9) implies that

$$
\left\langle I_{+}^{\prime}\left(u_{n}\right), u_{n}\right\rangle=\left\|u_{n}\right\|^{2}-\int_{\Omega} f_{+}\left(x, u_{n}(x)\right) u_{n} d x=o(1) .
$$

To complete our proof, we first need to verify that $\left\{u_{n}\right\}$ is bounded in $E$. Assume $\left\|u_{n}\right\| \rightarrow$ $+\infty$ as $n \rightarrow \infty$. Let

$$
s_{n}=\frac{2 \sqrt{|c|+1}}{\left\|u_{n}\right\|}, \quad w_{n}=s_{n} u_{n}=\frac{2 \sqrt{|c|+1} u_{n}}{\left\|u_{n}\right\|} .
$$

Since $\left\{w_{n}\right\}$ is bounded in $E$, it is possible to extract a subsequence (denoted also by $\left\{w_{n}\right\}$ ) such that

$$
\begin{aligned}
& w_{n} \rightarrow w_{0} \quad \text { in } E, \\
& w_{n}^{+} \rightarrow w_{0}^{+} \quad \text { in } L^{2}(\Omega), \\
& w_{n}^{+}(x) \rightarrow w_{0}^{+}(x) \quad \text { a.e. } x \in \Omega, \\
& \left|w_{n}^{+}(x)\right| \leq h(x) \quad \text { a.e. } x \in \Omega,
\end{aligned}
$$

where $w_{n}^{+}=\max \left\{w_{n}, 0\right\}, w_{0} \in E$ and $h \in L^{2}(\Omega)$.

We claim that if $\left\|u_{n}\right\| \rightarrow+\infty$ as $n \rightarrow+\infty$, then $w^{+}(x) \equiv 0$. In fact, we set $\Omega_{1}=\{x \in \Omega$ : $\left.w^{+}=0\right\}, \Omega_{2}=\left\{x \in \Omega: w^{+}>0\right\}$. Obviously, by (11), $u_{n}^{+} \rightarrow+\infty$ a.e. in $\Omega_{2}$; noticing condition 
$\left(\mathrm{H}_{3}\right)$, then, for any given $K>0$, we have

$$
\lim _{n \rightarrow+\infty} \frac{f\left(x, u_{n}^{+}\right)}{u_{n}^{+}}\left(w_{n}^{+}(x)\right)^{2} \geq K w^{+}(x)^{2} \quad \text { for a.e. } x \in \Omega_{2} .
$$

From (10), (11), and (12), we obtain

$$
\begin{aligned}
4(|c|+1) & =\lim _{n \rightarrow+\infty}\left\|w_{n}\right\|^{2}=\lim _{n \rightarrow+\infty} \int_{\Omega} \frac{f\left(x, u_{n}^{+}\right)}{u_{n}^{+}}\left(w_{n}^{+}\right)^{2} d x \\
& \geq \int_{\Omega_{2}} \lim _{n \rightarrow+\infty} \frac{f\left(x, u_{n}^{+}\right)}{u_{n}^{+}}\left(w_{n}^{+}\right)^{2} d x \geq K \int_{\Omega_{2}}\left(w^{+}\right)^{2} d x
\end{aligned}
$$

Noticing that $w^{+}>0$ in $\Omega_{2}$ and $K>0$ can be chosen large enough, so $\left|\Omega_{2}\right|=0$ and $w^{+} \equiv 0$ in $\Omega$. However, if $w^{+} \equiv 0$, then $\lim _{n \rightarrow+\infty} \int_{\Omega} F_{+}\left(x, w_{n}^{+}\right) d x=0$ and consequently

$$
I_{+}\left(w_{n}\right)=\frac{1}{2}\left\|w_{n}\right\|^{2}+o(1)=2(|c|+1)+o(1)
$$

By $\left\|u_{n}\right\| \rightarrow+\infty$ as $n \rightarrow+\infty$ and in view of (11), we observe that $s_{n} \rightarrow 0$, then it follows from Lemma 2.4 and (8) that

$$
I_{+}\left(w_{n}\right)=I_{+}\left(s_{n} u_{n}\right) \leq \frac{1+s_{n}^{2}}{2 n}+I_{+}\left(u_{n}\right) \rightarrow c \quad \text { as } n \rightarrow+\infty
$$

Clearly, (13) and (14) are contradictory. So $\left\{u_{n}\right\}$ is bounded in $E$.

Next, we prove that $\left\{u_{n}\right\}$ has a convergent subsequence. In fact, we can suppose that

$$
\begin{aligned}
& u_{n} \rightarrow u \quad \text { in } E, \\
& u_{n} \rightarrow u \quad \text { in } L^{q}(\Omega), \forall 1 \leq q<p^{*}, \\
& u_{n}(x) \rightarrow u(x) \quad \text { a.e. } x \in \Omega .
\end{aligned}
$$

Now, since $f$ has the improved subcritical growth on $\Omega$, for every $\varepsilon>0$, we can find a constant $C(\varepsilon)>0$ such that

$$
f_{+}(x, s) \leq C(\varepsilon)+\varepsilon|s|^{p^{*}-1}, \quad \forall(x, s) \in \Omega \times \mathbb{R},
$$

then

$$
\begin{aligned}
& \left|\int_{\Omega} f_{+}\left(x, u_{n}\right)\left(u_{n}-u\right) d x\right| \\
& \quad \leq C(\varepsilon) \int_{\Omega}\left|u_{n}-u\right| d x+\varepsilon \int_{\Omega}\left|u_{n}-u\right|\left|u_{n}\right|^{p^{*}-1} d x \\
& \quad \leq C(\varepsilon) \int_{\Omega}\left|u_{n}-u\right| d x+\varepsilon\left(\int_{\Omega}\left(\left|u_{n}\right|^{p^{*}-1}\right)^{\frac{p^{*}}{p^{*}-1}} d x\right)^{\frac{p^{*}-1}{p^{*}}}\left(\int_{\Omega}\left|u_{n}-u\right|^{p^{*}} d x\right)^{\frac{1}{p^{*}}} \\
& \quad \leq C(\varepsilon) \int_{\Omega}\left|u_{n}-u\right| d x+\varepsilon C(\Omega) .
\end{aligned}
$$

Similarly, since $u_{n} \rightarrow u$ in $E, \int_{\Omega}\left|u_{n}-u\right| d x \rightarrow 0$. Since $\varepsilon>0$ is arbitrary, we can conclude that

$$
\int_{\Omega}\left(f_{+}\left(x, u_{n}\right)-f_{+}(x, u)\right)\left(u_{n}-u\right) d x \rightarrow 0 \quad \text { as } n \rightarrow \infty .
$$


By (10), we have

$$
\left\langle I_{+}^{\prime}\left(u_{n}\right)-I_{+}^{\prime}(u),\left(u_{n}-u\right)\right\rangle \rightarrow 0 \quad \text { as } n \rightarrow \infty .
$$

From (15) and (16), we obtain

$$
\int_{\Omega}\left[\left|\Delta\left(u_{n}-u\right)\right|^{2}-c\left|\nabla\left(u_{n}-u\right)\right|^{2}\right] d x \rightarrow 0 \quad \text { as } n \rightarrow \infty .
$$

So we have $u_{n} \rightarrow u$ in $E$, which means that $I_{+}$satisfies $(C)_{c}$.

Next we prove that $I$ satisfies $(C)_{c}$. In fact, by $\left(\mathrm{H}_{4}\right)$, we have

$$
f(x, t) t-2 F(x, t) \geq f(x, s t) s t-2 F(x, s t)
$$

for $(x, t) \in \Omega \times \mathbb{R}$ and $s \in[0,1]$. By the proof of Lemma 2.1 in [19], we can similarly prove that $(C)_{c}$ sequence $\left\{u_{n}\right\}$ is bounded. The other part of the proof is similar to the case already proved and is omitted.

Lemma 2.6 Under the assumptions of Theorem $1.2, I_{+}$and I satisfy the $(C)_{c}$ condition.

Proof We only do the proof for $I_{+}$. Similar to the first part in the proof of Lemma 2.5, we easily know that $(C)_{c}$ sequence $\left\{u_{n}\right\}$ is bounded in $E$. Next, we prove that $\left\{u_{n}\right\}$ has a convergent subsequence. Without loss of generality, suppose that

$$
\begin{aligned}
& \left\|u_{n}\right\| \leq \beta_{0}, \\
& u_{n} \rightarrow u \quad \text { in } E, \\
& u_{n} \rightarrow u \quad \text { in } L^{q}(\Omega), \forall q \geq 1, \\
& u_{n}(x) \rightarrow u(x) \quad \text { a.e. } x \in \Omega .
\end{aligned}
$$

By the equivalence of the norm on $E$, we have

$$
\left\|u_{n}\right\|_{E} \leq C^{*}\left\|u_{n}\right\| .
$$

Let $\beta=C^{*} \beta_{0}$. Now, since $f_{+}$has the subcritical exponential growth (SCE) on $\Omega$, we can find a constant $C_{\beta}>0$ such that

$$
\left|f_{+}(x, t)\right| \leq C_{\beta} \exp \left(\frac{32 \pi^{2}}{2 \beta^{2}}|t|^{2}\right), \quad \forall(x, t) \in \Omega \times \mathbb{R} .
$$

Thus, by the Adams-type inequality (see Lemma 2.2),

$$
\begin{aligned}
& \left|\int_{\Omega} f_{+}\left(x, u_{n}\right)\left(u_{n}-u\right) d x\right| \\
& \quad \leq C\left(\int_{\Omega} \exp \left(\frac{32 \pi^{2}}{\beta^{2}}\left|u_{n}\right|^{2}\right) d x\right)^{\frac{1}{2}}\left|u_{n}-u\right|_{2} \\
& \quad \leq C\left(\int_{\Omega} \exp \left(\frac{32 \pi^{2}}{\beta^{2}}\left\|u_{n}\right\|_{E}^{2}\left|\frac{u_{n}}{\left\|u_{n}\right\|_{E}}\right|^{2}\right) d x\right)^{\frac{1}{2}}\left|u_{n}-u\right|_{2} \\
& \quad \leq C\left|u_{n}-u\right|_{2} \rightarrow 0 .
\end{aligned}
$$


Similar to the last part in the proof of Lemma 2.5, we have $u_{n} \rightarrow u$ in $E$, which means that $I_{+}$satisfies $(C)_{c}$.

\section{Computation of the critical groups}

It is well known that critical groups and Morse theory are the main tools in solving elliptic partial differential equations. Let us recall some results which will be used later. We refer the readers to the book [20] for more information on Morse theory.

Let $H$ be a Hilbert space and $I \in C^{1}(H, \mathbb{R})$ be a functional satisfying the (PS) condition (or $(C)_{c}$ condition), and $H_{q}(X, Y)$ be the $q$ th singular relative homology group with integer coefficients. Let $u_{0}$ be an isolated critical point of $I$ with $I\left(u_{0}\right)=c, c \in \mathbb{R}$, and let $U$ be a neighborhood of $u_{0}$. The group

$$
C_{q}\left(I, u_{0}\right):=H_{q}\left(I^{c} \cap U, I^{c} \cap U \backslash\left\{u_{0}\right\}\right), \quad q \in Z,
$$

is said to be the $q$ th critical group of $I$ at $u_{0}$, where $I^{c}=\{u \in H: I(u) \leq c\}$.

Let $K:=\left\{u \in H: I^{\prime}(u)=0\right\}$ be the set of critical points of $I$ and $a<\inf I(K)$, the critical groups of $I$ at infinity are formally defined by (see [21])

$$
C_{q}(I, \infty):=H_{q}\left(H, I^{a}\right), \quad q \in Z .
$$

For the convenience of our proof, we firstly prove two important propositions.

Proposition 3.1 If the assumptions of Theorem 1.1 (or Theorem 1.2) hold, then

$$
C_{q}(I, \infty)=0 \quad \text { for all integers } q \geq 0 .
$$

Proof Let $S^{\infty}=\{u \in E:\|u\|=1\}$ be the unit sphere in $E$ and $B^{\infty}=\{u \in E:\|u\| \leq 1\}$. By $\left(\mathrm{H}_{3}\right)$, for any $M>0$ there exists $c>0$, such that $F(x, t) \geq M t^{2}-c$, for $(x, t) \in \Omega \times \mathbb{R}$, which implies

$$
I(t u) \rightarrow-\infty \quad \text { as } t \rightarrow+\infty
$$

for any $u \in S^{\infty}$. Using $\left(\mathrm{H}_{4}\right)$, we have

$$
f(x, t) t-2 F(x, t) \geq 0 \quad \text { for }(x, t) \in \Omega \times \mathbb{R} .
$$

Choose

$$
a<\min \left\{\inf _{u \in B^{\infty}} I(u), 0\right\} .
$$

Then, for any $u \in S^{\infty}$, there exists $t>1$ such that $I(t u) \leq a$, that is,

$$
I(t u)=\frac{t^{2}}{2}-\int_{\Omega} F(x, t u) d x \leq a,
$$


which implies

$$
\begin{aligned}
\frac{d}{d t} I(t u) & =t-\int_{\Omega} f(x, t u) u d x \\
& \leq \frac{1}{t}(2 a)<0 \quad \text { by }(17) .
\end{aligned}
$$

The rest of the proof is similar to the proof of Lemma 2.6 in [19].

Proposition 3.2 If the assumptions of Theorem 1.1 (or Theorem 1.2) hold, then

$$
C_{q}(I, 0)=\delta_{q, 0} Z \text { for all integers } q \geq 0 .
$$

Proof By Lemma 2.1 (Lemma 2.3), $u=0$ is a local minimizer of $I$. So we have

$$
C_{q}(I, 0)=\delta_{q, 0} Z \quad \text { for all integers } q \geq 0 .
$$

\section{Proof of the main results}

Proofs of Theorem 1.1 and Theorem 1.2 By Lemma 2.1 (Lemma 2.3), Lemma 2.5 (Lemma 2.6), and Proposition 2.1, the functional $I_{+}$has a critical point $u_{1}$ satisfying $I_{+}\left(u_{1}\right) \geq \beta$. Since $I_{+}(0)=0, u_{1} \neq 0$ and by the maximum principle, we get $u_{1}>0$. Hence $u_{1}$ is a positive solution of the problem (1) and satisfies

$$
C_{1}\left(I_{+}, u_{1}\right) \neq 0, \quad u_{1}>0 .
$$

Using Lemma 3.1 in [22], we obtain

$$
C_{q}\left(I, u_{1}\right)=C_{q}\left(I_{C_{0}^{1}(\Omega)}, u_{1}\right)=C_{q}\left(\left.I_{+}\right|_{C_{0}^{1}(\Omega)}, u_{1}\right)=C_{q}\left(I_{+}, u_{1}\right)=\delta_{q 1} Z
$$

Similarly, we can obtain another negative critical point $u_{2}$ of $I$ satisfying

$$
C_{q}\left(I, u_{2}\right)=\delta_{q, 1} Z
$$

Now, from Proposition 3.2, we have

$$
C_{q}(I, 0)=\delta_{q, 0} Z
$$

On the other hand, from Proposition 3.1, we have

$$
C_{q}(I, \infty)=0 .
$$

Then from (19), (20), (21), (22), and the Morse relation, we have

$$
1+2 t=(1+t) Q(t)
$$

with $Q(t)=\sum_{q \geq 0} d_{q} t^{q}$ where $d_{q} \in \mathbb{N}=\{0,1,2, \ldots, n, \ldots\}$ for all $q \geq 0$. Then $d_{0}=d_{1}=1$ and so the right-hand side has a term $t^{2}$, a contradiction. This means that there is one more critical point of $I, u_{3} \notin\left\{0, u_{1}, u_{2}\right\}$. Then $u_{3} \in E$ is a nontrivial solution of problem (1). 
Competing interests

The authors declare that they have no competing interests.

\section{Authors' contributions}

The authors read and approved the final manuscript.

\section{Acknowledgements}

This study was supported by the National NSF (Grant No. 11101319) of China and Planned Projects for Postdoctoral Research Funds of Jiangsu Province (Grant No. 1301038C). The authors are very grateful for the reviewers' valuable comments and suggestions in improving this paper.

Received: 23 February 2015 Accepted: 18 June 2015 Published online: 09 July 2015

\section{References}

1. Lazer, AC, Mckenna, PJ: Large amplitude periodic oscillation in suspension bridges: some new connections with nonlinear analysis. SIAM Rev. 32, 537-578 (1990)

2. Lazer, AC, Mckenna, PJ: Global bifurcation and a theorem of Tarantello. J. Math. Anal. Appl. 181, $648-655$ (1994)

3. Tarantello, G: A note on a semilinear elliptic problem. Differ. Integral Equ. 5, 561-566 (1992)

4. Micheletti, AM, Pistoia, A: Multiplicity solutions for a fourth order semilinear elliptic problems. Nonlinear Anal. TMA 31, 895-908 (1998)

5. Xu, GX, Zhang, JH: Existence results for some fourth-order nonlinear elliptic problems of local superlinearity and sublinearity. J. Math. Anal. Appl. 281, 633-640 (2003)

6. Zhang, JH: Existence results for some fourth-order nonlinear elliptic problems. Nonlinear Anal. TMA 45, 29-36 (2001)

7. Zhang, JH, Li, SJ: Multiple nontrivial solutions for some fourth-order semilinear elliptic problems. Nonlinear Anal. TMA 60, 221-230 (2005)

8. An, YK, Liu, RY: Existence of nontrivial solutions of an asymptotically linear fourth-order elliptic equation. Nonlinear Anal. TMA 68, 3325-3331 (2008)

9. Liu, Y, Wang, ZP: Biharmonic equations with asymptotically linear nonlinearities. Acta Math. Sci. 27, $549-560$ (2007)

10. Zhang, J, Wei, ZL: Infinitely many nontrivial solutions for a class of biharmonic equations via fountain theorems. Nonlinear Anal. TMA 74, 7474-7485 (2011)

11. Liu, XQ, Huang, YS: On sign-changing solution for a fourth-order asymptotically linear elliptic problem. Nonlinear Anal. TMA 72, 2271-2276 (2010)

12. Zhou, JW, Wu, X: Sign-changing solutions for some fourth-order nonlinear elliptic problems. J. Math. Anal. Appl. 342, 542-558 (2008)

13. Lam, N, Lu, GZ: N-Laplacian equations in $\mathbb{R}^{N}$ with subcritical and critical growth without the Ambrosetti-Rabinowitz condition. Adv. Nonlinear Stud. 13, 289-308 (2013)

14. Liu, ZL, Wang, ZQ: On the Ambrosetti-Rabinowitz superlinear condition. Adv. Nonlinear Stud. 4, 563-574 (2004)

15. Pei, RC, Zhang, JH: Biharmonic equations with improved subcritical polynomial growth and subcritical exponential growth. Bound. Value Probl. 2014, 162 (2014)

16. Ruf, B, Sani, F: Sharp Adams-type inequalities in $\mathbb{R}^{N}$. Trans. Am. Math. Soc. 365, 645-670 (2013)

17. Costa, DG, Miyagaki, OH: Nontrivial solutions for perturbations of the $p$-Laplacian on unbounded domains. J. Math. Anal. Appl. 193, 737-755 (1995)

18. Zhou, HS: Existence of asymptotically linear Dirichlet problem. Nonlinear Anal. TMA 44, 909-918 (2001)

19. Sun, JJ, Ma, SW: Nontrivial solutions for Kirchhoff type equations via Morse theory. Discrete Contin. Dyn. Syst. 13, 483-494 (2014)

20. Chang, KC: Infinite Dimensional Morse Theory and Multiple Solutions Problems. Birkhäuser, Boston (1993)

21. Bartsch, T, Li, SJ: Critical point theory for asymptotically quadratic functionals and applications to problems with resonance. Nonlinear Anal. 28, 419-441 (1997)

22. Qian, AX, Li, SJ: Multiple solutions for a fourth-order asymptotically linear elliptic problem. Acta Math. Sin. 22, 1121-1126 (2006)

\section{Submit your manuscript to a SpringerOpen ${ }^{\circ}$ journal and benefit from:}

- Convenient online submission

- Rigorous peer review

- Immediate publication on acceptance

- Open access: articles freely available online

- High visibility within the field

- Retaining the copyright to your article 